Reprod. Nutr. Dévelop., 1982, 22 (2), 363-369.

\title{
Effets de la prise alimentaire sur la sécrétion biliaire chez le lapin
}

\author{
R. JIMÉNEZ, F. LISBONA, A. ESTELLER, María Abdona LÓPEZ
}

Département de Physiologie animale, Faculté des Sciences, Université de Granada, Espagne.

Summary. Effects of food intake on biliary response in the rabbit.

Basal biliary secretion, biliary response to a prolonged period of feeding with or without bile into the intestine, and the choleretic efficacy of bile after total interruption of enterohepatic circulation of bile salts (EHC of BS) have been investigated in the rabbit. The bile flow increase after intake of food only when bile was returned into the intestine. The bile flow, bile salt concentration and bile salt output dropped after the EHC of BS was interrupted, in spite of feeding conditions; when bile was reinfused, all the parameters returned to the previous levels.

Introduction.

Plusieurs travaux ont déjà été publiés sur la sécrétion biliaire postprandiale chez le rat (Shaw et Heath, 1972; Vonk, Van Doorn et Strubbe, 1978), le chien (Austin et al., 1978), le porc (Juste, Corring et Breant, 1978), les primates (Strasberg, Siminovitch et Ilson, 1974) et autres espèces. Les données relatives à la sécrétion biliaire chez le lapin anesthésié sont aussi abondantes (Esteller, Lopez et Murillo, 1977 ; Klaassen, 1973 ; Rutishauser et Stone, 1975 ; Scratcherd, 1965). Toutefois, les informations disponibles sur la sécrétion biliaire chez l'animal éveillé sont plus restreintes (Esteller, Jiménez et Lopez, 1981 ; Shaw et Heath, 1974) bien que le lapin soit un animal expérimental très utilisé. Dans le présent travail nous avons recherché l'importance de la réponse à l'alimentation en continu de quelques paramètres de la sécrétion biliaire (débit, concentration en sels biliaires et quantité de sels biliaires) après un jeûne de $11 \mathrm{~h}$. A notre avis, la prise de nourriture joue un rôle important sur la formation et la sécrétion de la bile chez cet animal qui mange pratiquement de façon continue et présente un débit de bile élevé. 


\section{Matériel et méthodes.}

Animaux et régime. - Un nombre total de 30 lapins de race Castellana, de poids vif compris entre 2,2 et $2,8 \mathrm{~kg}$, sont utilisés. Les animaux reçoivent ad libitum un régime commercial ( $\left.{ }^{1}\right)$ contenant 14,5 p. 100 de protéines au minimum et 2 p. 100 de lipides. Ils sont munis d'un collier pour empêcher la cæecotrophie pendant les périodes expérimentales.

Technique chirurgicale. - Dans le but d'obtenir la bile directement du canal cholédoque pendant de longues périodes, les animaux sont porteurs de fistules biliaires chroniques. L'anesthésique employé est le pentobarbital sodique à la dosse de $20 \mathrm{mg} / \mathrm{kg}$ par voie intraveineuse. Deux cathéters en silastic médical $\varnothing$ intérieur : $1,97 \mathrm{~mm} ; \varnothing$ extérieur : $3,16 \mathrm{~mm}$ ) sont placés dans le cholédoque, l'un $a$, en direction du foie pour recueillir la bile, l'autre $b$, en direction du duodénum pour la réintroduire. Les cathéters sont extériorisés à travers la paroi abdominale droite et connectés à l'extérieur.

Soins aux animaux et protocole expérimental. - L'expérimentation a débuté après une période postopératoire de 4 à 5 jours, pendant laquelle les animaux sont soumis à un traitement anti-infectieux.

Nous avons réalisé 3 types d'expériences désignées respectivement par $A, B$ et $C$, toujours après une période de jeûne de $12 \mathrm{~h}$.

Expérience $A$ : Pendant $11 \mathrm{~h}$ les animaux sont maintenus en conditions basales (à jeun et avec une $\mathrm{CEH}\left({ }^{2}\right)$ des SB $\left({ }^{2}\right)$ intacte, grâce au retour de la bile dans le bascholédoque par le cathéter $b$ ).

Expérience $B$ : Elle dure $11 \mathrm{~h}$; la $\mathrm{CEH}$ des SB demeure intacte, par retour de la bile dans le bas-cholédoque, et l'aliment est présenté aux animaux pendant $7 \mathrm{~h}$, de la $5^{\mathrm{e}}$ à la $11^{\mathrm{e}}$ heure. L'alimentation est donnée ad libitum mais la prise de nourriture est contrôlée toutes les heures.

Expérience $C$ : Elle s'étend sur une durée de $15 \mathrm{~h}$, la $\mathrm{CEH}$ des SB étant interrompue pendant $7 \mathrm{~h}$, de la $5^{\mathrm{e}}$ à la $11^{\mathrm{e}}$ heure, par dérivation complète de la bile vers l'extérieur. L'alimentation, donnée ad libitum, est présentée aux animaux pendant la période d'interruption de la $\mathrm{CEH}$ des $\mathrm{SB}$ et l'ingestion est également contrôlée toutes les heures.

Pendant les essais la bile est recueillie en continu dans des poches de polyéthylène sur des périodes successives de $60 \mathrm{~min}$. Le volume horaire de bile est déterminé par pesée. Une partie aliquote $(5 \mathrm{p} .100)$ est stockée pour analyses à $-20^{\circ} \mathrm{C}$, et le reste est réintroduit en 4 fractions toutes les $15 \mathrm{~min}$. Le débit biliaire est exprimé en $\mu \mathrm{l} / \mathrm{min}$.

(1) Régime pour lapin R-02, Sanders.

(2) $\mathrm{CEH}$ : circulation entérohépatique ; $\mathrm{SB}$ : sels biliaires. 
Analyses chimiques. - La concentration des SB totaux dans la bile est déterminée par colorimétrie (Levin, Johnston et Boyle, 1961) et exprimée en mEq d'acide cholique/l.

Traitement statistique. - Le test de $\mathrm{t}$ de Student a été utilisé pour l'analyse statistique des résultats.

\section{Résultats.}

L'ensemble des résultats obtenus dans les expériences $A, B$ et $C$, chez les animaux à jeun dont la CEH des SB est conservée (période basale) montre que, dans ces conditions, le débit biliaire basal est de $69,4 \pm 1,8 \mu \mathrm{l} / \mathrm{min}$. (n $=170$ ) et la concentration en $S B$ de la bile est $21,4 \pm 1,1 \mathrm{mEq} / \mathrm{l}$ ( $\mathrm{n}=60$ ).

Dans l'expérience $A$, considérée comme expérience témoin, le débit biliaire, la concentration des SB et la quantité de SB sécrétés (excrétion des SB) oscillent respectivement autour de $70 \mu \mathrm{l} / \mathrm{min}$., $18 \mathrm{mEq} / \mathrm{l}$ et $1,3 \mu \mathrm{Eq} / \mathrm{min}$. Les variations autour de ces valeurs moyennes sont négligeables pour le débit biliaire (fig. 1a), soit de 12 p. 100 et 25 p. 100 environ respectivement pour les concentrations et les quantités de SB (fig. 1b). Ces variations ne sont pas statistiquement significatives.

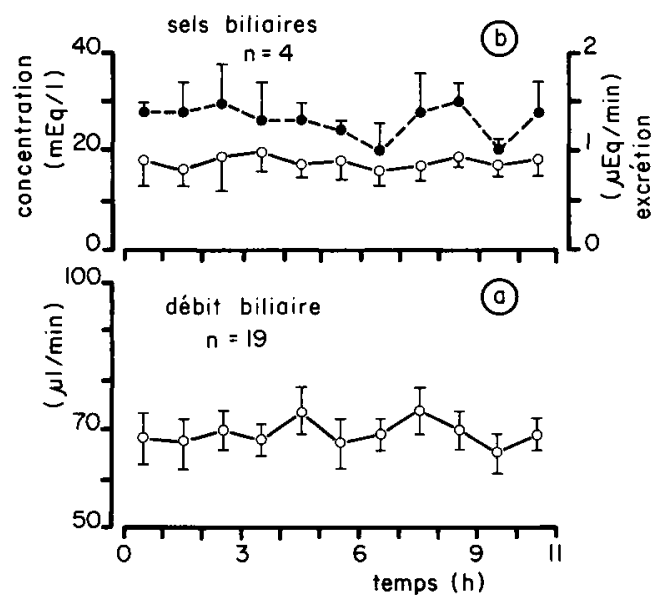

FIG. 1. - Débit biliaire, concentration en sels biliaires de la bile et excrétion des sels biliaires en fonction du temps chez les animaux témoins (expérience A). Valeurs moyennes \pm écartstypes de la moyenne. $\mathrm{O} \longrightarrow \mathrm{O}$ Concentration en sels biliaires ; biliaires ; $\mathbf{n}=$ nombre de données.

Dans l'expérience B, chez les animaux dont la CEH des SB est maintenue intacte, l'ingestion d'aliment pendant $7 \mathrm{~h}$ provoque une série de modifications illustrées par la figure 2 . Le débit biliaire augmente, bien que non significativement, dès la première heure consécutive au début de la période d'alimentation 
(fig. 2a). Cependant, dans quelques cas (4 observations), cette augmentation ne commence qu'à la $2^{\mathrm{e}}$ heure. A la $7^{\mathrm{e}}$ heure le débit biliaire $(105 \pm 8 \mu \mathrm{l} / \mathrm{min}$.) est significativement supérieur de 40 p. 100 en moyenne au débit observé durant la période préprandiale $(75 \pm 7 \mu \mathrm{l} / \mathrm{min}$.). Cette augmentation peut atteindre 300 p. 100 chez certains animaux (3 observations). La quantité de SB sécrétés s'élève parallèlement au débit biliaire, atteignant un niveau maximal à la fin de l'expérience: 37 p. 100 par rapport aux valeurs préprandiales (fig. 2c); cet accroissement devient significatif dans la $7^{\mathrm{e}}$ heure après le début de l'ingestion d'aliment. En revanche, la concentration des SB dans la bile diminue légèrement, quoique non significativement en période d'alimentation (15 p. 100 environ par rapport aux valeurs préprandiales, fig. $2 \mathrm{~b}$ ).

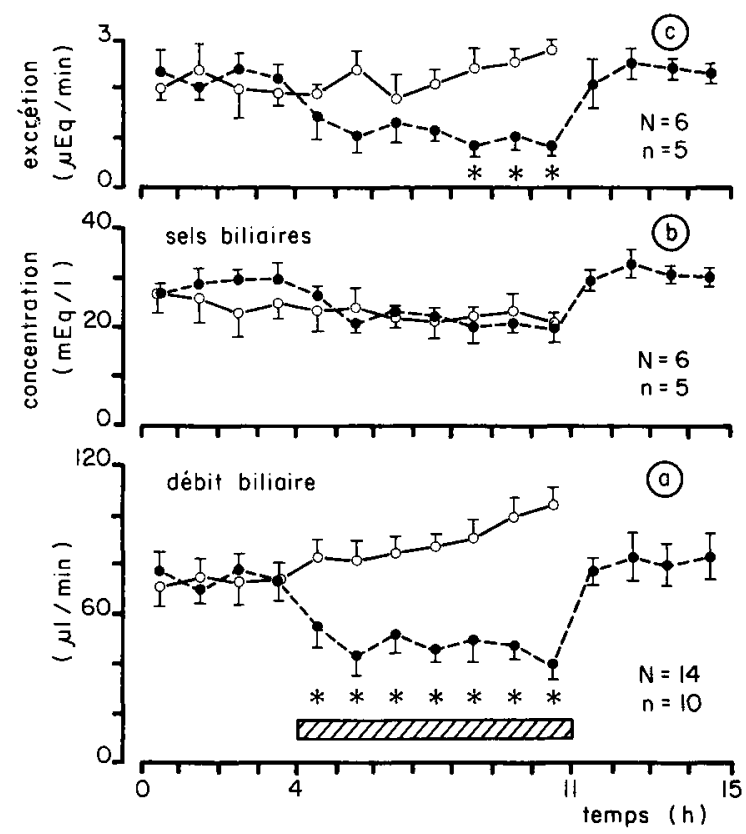

FIG. 2. - Effets de la prise de nourriture sur le débit biliaire, la concentration en sels biliaires de la bile et l'excrétion des se/s biliaires en fonction du temps.

$\mathrm{O}-\mathrm{O}$ Avec la circulation entérohépatique des sels biliaires intacte (expérience $B$ ), $(N)$ : - - - - Avec la circulation entérohépatique des sels biliaires interrompue (expérience C), (n). $\mathrm{N}, \mathrm{n}=$ nombre de données $;^{*}=\mathrm{p}<0.001$. ZZZJ : Prise de nourriture, valable pour les graphiques $a$, $b$, et $c$.

Dans l'expérience $C$, les animaux alimentés selon le même schéma que dans l'expérience $B$, sont soumis à une interruption du recyclage entérohépatique des SB. Dans ces conditions le débit biliaire diminue significativement dès la $2^{\mathrm{e}}$ heure d'interruption jusqu'à une valeur minimale de $40 \mu \mathrm{l} / \mathrm{min}$., qui représente environ 50 p. 100 de la valeur initiale (fig. 2a). Une diminution significative de la concentration des SB et de l'excrétion des SB est aussi observée (fig. $2 b$ et $c$ ) ; après cette chute, les deux paramètres se stabilisent, dès la $5^{\mathrm{e}}$ heure $\mathrm{d}^{\prime}$ interruption, à 
des valeurs d'environ 30 et 35 p. 100 respectivement des valeurs initiales. Si dans ces conditions la $\mathrm{CEH}$ des $\mathrm{SB}$ est rétablie par réintroduction de la bile au niveau cholédoque (fig. $2,12^{\mathrm{e}}$ à $15^{\mathrm{e}}$ heure) le débit biliaire, la concentration de la bile en $\mathrm{SB}$ et la quantité de SB sécrétés reviennent aux valeurs trouvées avant I'interruption de la $\mathrm{CEH}$ des SB.

La prise de nourriture, contrôlée pendant la période d'alimentation dans les expériences $B$ et $C$, est très variable pour un même animal et d'une heure à l'autre. Pour l'ensemble de toutes les observations ( $n=168$ ), l'ingestion est de $14,7 \pm 8,5 \mathrm{~g} / \mathrm{h}$.

\section{Discussion.}

Si l'on exprime les résultats par kg de poids corporel, le débit biliaire dans les conditions basales chez le lapin éveillé est similaire $(30 \mu \mathrm{l} / \mathrm{min} . / \mathrm{kg})$, à celui du porc (Juste, Corring et Breant, 1979), mais est supérieur à celui du mouton (Heath, Caple et Redinger, 1970) et du chien (Wheeler et Ramos, 1960). Par contre, il est moins important que celui observé par d'autres auteurs chez le lapin anesthésié (Esteller, Lopez et Murillo, 1977 ; Klaassen, 1973 ; Shaw et Heath, 1974). Nous attribuons cette différence à l'effet des anesthésiques sur la sécrétion biliaire (Berthelot et al., 1970). La concentration de la bile en SB pendant la période basale $(21,4 \pm 1,1 \mathrm{mEq} / \mathrm{l})$ est environ la moitié de celle observée chez le porc (Juste, Corring et Breant, 1979), mais est plus importante que celle décrite, chez le lapin anesthésié, par Rutishauser et Stone (1975).

Les variations du débit biliaire et de la concentration de la bile en SB observées chez les animaux dont la CEH des SB est conservée pendant $11 \mathrm{~h}$ de jeûne, sont en accord avec celles trouvées dans la littérature pour d'autres espèces (Austin et al., 1978 ; Vonk, Van Doorn et Strubbe, 1978).

L'augmentation du débit biliaire en réponse à l'ingestion d'aliment pendant une période prolongée $(7 \mathrm{~h})$, chez les animaux dont la $\mathrm{CEH}$ des SB demeure intacte, confirment celles rapportées par Esteller, Jiménez et Lopez (1981) chez le lapin, Austin et al. (1978) chez le chien et Vonk, Van Doorn et Strubbe (1978) chez le rat. Ces résultats diffèrent de ceux obtenus par Shaw et Heath (1972) chez le rat, ces auteurs n'ayant observé aucune modification dans le débit, en réponse à l'alimentation avec une $\mathrm{CEH}$ des SB intacte. Malgré la diminution de la concentration de la bile en SB observée en réponse à la prise de nourriture, l'excrétion biliaire des SB augmente simultanément en raison de la forte stimulation du débit biliaire postprandial. Ces résultats permettent de penser que la vésicule biliaire chez le lapin a une fonction peu importante dans la réponse à la prise de nourriture, ce qui est en accord, d'une part avec le faible pouvoir de concentration de la bile au niveau de la vésicule biliaire chez cette espèce (Esteller et al., 1975), et d'autre part avec les résultats obtenus chez l'animal cholécystectomisé (Esteller, Jiménez et Lopez, 1981).

Inversement, quand la nourriture est donnée à l'animal dont la CEH des SB est interrompue, on n'observe pas d'augmentation du débit biliaire, mais au contraire une diminution significative par comparaison avec les valeurs obtenues chez 
les animaux qui ont conservé intacte une CEH des SB et celles observées dans les expériences de contrôle. Ces résultats sont en désaccord avec ceux obtenus d'une part par Nahrwold et Grossman (1967) sur le chien cholécystectomisé et d'autre part par Shaw et Heath (1972) sur le rat.

Toutes ces données nous conduisent à penser que chez le lapin : a) Bien que le débit biliaire soit élevé, celui-ci augmente en réponse à la prise de nourriture, même si l'aliment est présenté pendant de longues périodes. $b$ ) L'effet hypocholerétique de l'interruption de la $\mathrm{CEH}$ des SB sur le débit et la concentration de la bile en SB est plus important que l'effet hypercholerétique de la prise de nourriture. c) La diminution importante de la concentration en SB de la bile chez les animaux où la $\mathrm{CEH}$ des $\mathrm{SB}$ est interrompue et qui sont nourris, est due à ce que dans ce cas s'additionnent les effets de l'alimentation et de l'interruption de la $\mathrm{CEH}$ des SB. $d$ ) Le pouvoir cholerétique de la bile elle-même, qui permet notamment un rapide retour des valeurs du débit biliaire et de la concentration en SB de la bile à un niveau basal, après interruption de la CEH des SB pendant $7 \mathrm{~h}$, apparaît plus important que l'effet de stimulation de l'alimentation, même si cette dernière est prolongée sur une période identique.

Reçu en juillet 1981. Accepté en octobre 1981.

\section{Références}

AUSTIN M. I., JOHNSON S. M., SHIRES G. I., JONES R. S., 1978. The effect of feeding on the bile salt-independent canalicular secretion in dogs. Am. J. Surg., 135, 36-39.

BERTHELOT P., ERLINGER S., DHUMEAUX D., PAVAUX A. M., 1970. Mechanism of phenobarbital induced hypercholeresis in the rat. Am. J. Physiol., 219, 809-813.

ESTELLER A., HIGUERA M., LOPEZ M. A., MURILLO A., ZAMORA S., 1975. Capacidad concentradora de la vesícula biliar : Estudio comparativo en algunas espécies de vertebrados. Rev. esp. Fisiol., 31, 91-94.

ESTELLER A., JIMÉNEZ R., LOPEZ M. A., 1981. Biliary secretion in conscious rabbits : Role of enterohepatic circulation of bile salts and gall bladder. Quart. J. exp. Physiol., 66, 349-357.

ESTELLER A., LOPEZ M. A., MURILLO A., 1977. The effect of secretin and cholecystokininpancreozymin on the secretion of bile in the anaesthetized rabbit. Quart. J. exp. Physiol., 62, 353-359.

HEATH T., CAPLE I. W., REDINGER P. M., 1970. Effect of enterohepatic circulation of bile salts and lipids in sheep. Quart. J. exp. Physiol., 55, 93-103.

JONES R. S., GROSSMAN M. I., 1969. The choleretic response to feeding in dogs. Proc. Soc. exp. Biol. Med., 132, 708-711.

JUSTE C., CORRING T., BREANT Ph., 1979. Excrétion biliaire chez le porc : niveau et réponse au repas. Ann. Biol. anim. Bioch. Biophys., 19, 79-90.

KLAASSEN C. D., 1973. Bile flow and bile composition during bile acids depletion and administration. Can. J. Physiol. Pharmacol., 52, 334-348.

LEVIN S. J., JOHNSTON C. G., BOYLE A. J., 1961. Spectrophotometric determination of several bile acids as conjugated. Analyt. Chem., 33, 1407-1411.

NAHRWOLD D. L., GROSSMAN M. I., 1967. Secretion of bile in response to food with and without bile in the intestine. Gastroenterology, 53, 11-16.

RUTISHAUSER S. C. B., STONE S. L., 1975. Aspects of bile secretion in the rabbit. J. Physiol., 245, 567-582. 
SCRATCHERD T., 1965. Electrolyte composition and control of biliary secretion in the cat and rabbit, 515-519. In TAYLOR W., Biliary system. Blackwell, Oxford.

SHAW H. M., HEATH T. J., 1972. The significance of hormones, bile salts and feeding in the regulation of bile and other digestive secretion in the rat. Aust. J. biol. Sci., 25, 147154.

SHAW H. M., HEATH T. J., 1974. Regulation of bile formation in rabbits and guinea pigs. Quart. J. exp. Physiol., 59, 93-102.

STRASBERG S. M., SIMINOVITCH R. G., ILSON R. G., 1974 . Bile production in fasted and fed primates. Ann. Surg., 180, 356-363.

VONK R. J., VAN DOORN A. B. D., STRUBBE J. H., 1978. Bile secretion and bile composition in the freely moving, unanaesthetized rat with a permanent biliary drainage : influence of food intake on bile flow. Clin. Sci. mol. Med., 55, 253-259.

WHEELER H. O., RAMOS O. L., 1960. Determinants of the flow and composition of bile in the unanaesthetized dog during constant infusion of sodium taucholate. $J$. clin. Invest., 39, 161-170. 